\title{
Narrative review in the current role of angiotensin receptor- neprilysin inhibitors
}

\author{
Jose B. Cruz Rodriguez ${ }^{1}$, Cameron $\mathrm{Cu}^{2}$, Tariq Siddiqui ${ }^{1}$ \\ ${ }^{1}$ Division of Cardiovascular Diseases, ${ }^{2}$ Department of Internal Medicine, Texas Tech University Health Science Center El Paso, El Paso, TX, USA \\ Contributions: (I) Conception and design: All authors; (II) Administrative support: JB Cruz Rodriguez, T Siddiqui; (III) Provision of study materials \\ or patients: JB Cruz Rodriguez, C Cu; (IV) Collection and assembly of data: All authors; (V) Data analysis and interpretation: All authors; (VI) \\ Manuscript writing: All authors; (VII) Final approval of manuscript: All authors. \\ Correspondence to: Jose B. Cruz Rodriguez. Division of Cardiovascular Diseases, Texas Tech University Health Science Center El Paso. 4800 Alberta \\ Avenue, El Paso, TX 79905, USA. Email: Benjamin.cruz@ttuhsc.edu.
}

\begin{abstract}
Heart failure (HF) accounts for a tremendous burden on health care systems and the society. Since the landmark PARADIGM-HF trial, sacubitril/valsartan, the first in the class of angiotensin receptor neprilysin inhibitor (ARNI) showed superiority to enalapril in patients with HF with reduced ejection fraction (HFrEF). We performed a narrative literature review, hand-searched the reference lists of included articles and relevant reviews. Inhibition of neprilysin increases bradykinin, natriuretic peptides and adrenomedullin levels counteract the neurohormal activation that leads to sodium retention, vasoconstriction, and cardiac remodeling. In PARADIGM-HF the primary outcome of CV death or HF hospitalization was reduced $20 \%$ in the ARNI group (HR $0.80, \mathrm{P}<0.001$ ) similar to mortality due to cardiovascular cause (HR 0.80, $\mathrm{P}<0.001$ ) in patients with $\mathrm{HFrEF}$, rendering a number needed to treat of 21 patients. This effect was consistent across subgroups. The safety of starting ARNI inpatient once the acute decompensation of HF is stabilized was demonstrated in PIONEER-HF trial. With willingness-topay thresholds commonly acceptable in the United States, sacubitril/valsartan is likely to be cost effective, which might not be in other health systems. Although its safety has been reassured in some clinical trials, common side effects are hypotension, worsening kidney function, hyperkalemia and angioedema. In HFpEF (PARAGON-HF), sacubitril/valsartan showed decrease in the level of the cardiac biomarkers, with improve functional NYHA and decrease in hospitalizations, predominately in women and patients with borderline ejection fraction. Some ongoing studies aim to demonstrate the effects of ARNI in acute coronary syndrome, stable ischemic heart disease, advanced HF, mitral regurgitation, aortic impedance and pulmonary hypertension. In conclusion, sacubitril/valsartan has proven to be an effective addition to the HFrEF arsenal, with safety comparable to current standard of care. In HFpEF, it improves quality of life, particularly in women and in patients with borderline ejection fraction, with no effect on mortality.
\end{abstract}

Keywords: Sacubitril; sacubitril/valsartan; neprilysin; LCZ696; angiotensin receptor neprilysin inhibitor (ARNI)

Submitted May 19, 2020. Accepted for publication Jul 30, 2020.

doi: $10.21037 /$ atm-20-4038

View this article at: http://dx.doi.org/10.21037/atm-20-4038

\section{Introduction}

Chronic heart failure (HF) accounts for $2 \%$ of health care costs in developed countries. Almost 34.8 billion dollars spent on this, make it the largest expenditure entity in the USA. It is estimated to rise by $127 \%$ by 2030 (1-3).
With the landmark PARADIGM-HF trial in 2014 (4), the management of HF advanced into a new era when LCZ696, now known as sacubitril/valsartan became the novel therapy and first in its class of angiotensin receptor neprilysin inhibitor (ARNI). It was compared with enalapril in addition to recommended treatment in chronic HF with 
class II-IV HF with reduced ejection fraction (HFrEF) of $40 \%$ or less.

Due to extensive literature on the only approved ARNI, we performed a narrative literature review on the topic aiming to clarify its current role in the spectrum of cardiovascular disease. We searched the Cochrane Central Register of Controlled Trials (CENTRAL), Cumulative Index to Nursing and Allied Health Literature (CINAHL), Embase, and PubMed databases through April 30th, 2020, with no restrictions on language. Key words of sacubitril, sacubitril/valsartan, neprilysin, LCZ696 and ARNi were utilized, crossed with HF. Randomized clinical trials, large prospective studies, systematic reviews and metanalysis were included. We hand-searched the reference lists of included articles and relevant reviews. We present the following article in accordance with the NARRATIVE REVIEW reporting checklist (available at http://dx.doi.org/10.21037/ atm-20-4038).

\section{Pharmacology of ARNI}

The natriuretic peptide system regulates volume, sodium levels, vasodilation, among other integral compensatory processes in HF. Neprilysin degrades beneficial natriuretic proteins namely ANP (atrial natriuretic peptide), BNP (B-type natriuretic peptide), and CNP (C-type natriuretic peptide). NT-proBNP is not a substrate for neprilysin, hence not being degraded (5). The protective and compensatory mechanisms of natriuretic peptides in $\mathrm{HF}$ seems to be deficient in early HF (6).

Cumulatively, the natriuretic peptide system has beneficial effects by inhibition of renin release, sympathetic nervous system, antidiuretic hormone release, lusitropic properties, enhancing vagal tone, and prevention of cardiac hypertrophy and fibrosis (7). Inhibition of neprilysin increases bradykinin, natriuretic peptides and adrenomedullin levels, counteracting the neurohormonal activation that leads to sodium retention, vasoconstriction, and cardiac remodeling $(8,9)$. Because neprilysin inhibition simultaneously increases angiotensin II levels, concurrent renin-angiotensin-aldosterone system (RAAS) inhibition is necessary $(10,11)$.

Another neprilysin inhibitor, omapatrilat, showed early promise for hypertension and HF. Phase II trials returned good results, attributed to its inhibition of neprilysin, ACE, and aminopeptidase (12). Unfortunately, the phase III OVERTURE trial (13), failed to achieve its primary endpoint of death or HF hospitalization. Significant results were found in post-hoc analysis with a more contemporary definition of HF hospitalization, but further investigation of omapatrilat was stopped due to an unacceptable level of angioedema (14).

\section{ARNIs in HFrEF}

Angiotensin converting enzyme (ACE) inhibitors had long been the cornerstone for HF therapy due to their numerous benefits including mortality reduction seen in the SOLVD-T, SOLVD-P, and CONSENSUS trials (15-17). PARADIGM-HF (4) involved 8,399 patients from 47 countries with left ventricular ejection fraction (LVEF) $\leq 40 \%$, NYHA II-IV and on at least 1 month of a stable dose of beta blocker and ACEi [or angiotensin receptor blocker (ARB)]. Approximately one-half of participants were also taking a mineralocorticoid receptor antagonist (MRA). Patients with hypotension, glomerular filtration rate (GFR) $<30 \mathrm{~mL} / \mathrm{min} / \mathrm{m}^{2}$, hyperkalemia and a history of angioedema were excluded from the trial $(18,19)$. There was a run-in period of 4-6 weeks before randomization, assuring that patients tolerated the target doses and both medications were held the day before randomization to minimized risk of angioedema.

The primary outcome of $\mathrm{CV}$ death or HF hospitalization was less in the in sacubitril/valsartan group, $21.8 \%$ compared to $26.5 \%$ in the enalapril group, HR 0.80 (95\% CI: $0.73-0.87 ; \mathrm{P}<0.001)$, as was the secondary outcome of mortality due to cardiovascular cause, from $16.5 \%$ to 13.3\%, HR: 0.80 (95\% CI: 0.71-0.89; $\mathrm{P}<0.001$ ). This conferred a number needed to treat of 21 patients to prevent one primary event. The secondary endpoints of death from any cause, $19.8 \%$ in sacubitril/valsartan group and $17.0 \%$ in enalapril group; HR 0.84 (95\% CI: 0.76-0.93; $\mathrm{P}<0.001)$ and improved symptoms with less decline in Kansas City CM Questionnaire (KCCQ) scores ( $\mathrm{P}=0.001)$ in the sacubitril/ valsartan group.

During the run-in portion, the enalapril group, had more participants withdraw due to adverse effects like cough, decline in renal function (worsening of $>25 \%$ in GFR, later increased by protocol to $>35 \%$ ), and progression to ESRD. In the sacubitril/valsartan group, there was more angioedema $(n=19$ versus $n=10$, none causing airway compromise) and hypotension. The trial was ended early after the prespecified limit for "overwhelming benefit" was reached (4). On further analysis of PARADIGM-HF, it was found that less participants in the sacubitril/valsartan arm needed dose increases in HF therapy (HR 0.84, 95\% CI: 
0.74-0.94) or emergency room care for HF decompensation (HR 0.66, 95\% CI: 0.52-0.85). The sacubitril/valsartan arm was less likely to require intensive care or IV inotropes, receive less $\mathrm{HF}$ device placement or cardiac transplant, and had lower NT pro-BNP and troponin levels compared to the enalapril group (20).

The benefits of sacubitril/valsartan over ACE inhibitor were significant regardless if $\mathrm{HFrEF}$ was optimized on guideline directed medical therapy or not. This included those with and without implanted defibrillator, on MRA, and on varying doses of beta-blocker (21).

Single center studies and reports have shown that ARNI improves LVEF, LV end systolic and end diastolic volumes, less mitral regurgitation and parameters of diastolic dysfunction (E/A ratio, diastolic filling time, restrictive mitral filling pattern) in a dose dependent fashion $(22,23)$.

The PIONEER-HF trial (24), sought to evaluate ARNI in hospitalized HFrEF patients. Patients included had a NT-proBNP $\geq 1,600 \mathrm{pg} / \mathrm{mL}$ or $\mathrm{BNP} \geq 400 \mathrm{pg} / \mathrm{mL}$ and LVEF $<40 \%$. To be randomized and allocated to either the ARNI or enalapril group, patients had to first be stabilized from acute decompensated HF, (systolic blood pressure greater than $100 \mathrm{mmHg}$ and no intravenous vasodilator or intravenous diuretic). Starting ARNI during hospitalization resulted in larger decreases in NT-proBNP compared with the enalapril group. More importantly, occurrence of angioedema, hyperkalemia, symptomatic hypotension, and renal dysfunction were similar between with ARNI and enalapril arms. These findings support the use of sacubitril/ valsartan in the setting of acute stabilized HF, and initiating therapy in the index hospitalization.

Meta-analysis of IMPRESS (12), OVERTURE (13) and PARADIGM-HF trials (4), further supports the use of ARNI over ACE inhibitor to decrease mortality, pooled HR 0.88 (95\% CI: 0.80-0.98) and decreasing composite death or HF hospitalization, pooled HR 0.86 (95\% CI: 0.76-0.97) (25).

\section{ARNIs in HF with preserved ejection fraction}

Neprilysin inhibition may be of particular benefit in positively altering the course of difficult to treat $\mathrm{HF}$ with preserved ejection fraction (HFpEF) (26). Increased natriuretic peptides from neprilysin inhibition can modulate cyclic GMP pathways, and possibly reduce the stiffness of myocardium by further phosphorylating rigid titin isoforms (27). Responsiveness to endothelium derived adrenomedullin was improved in animal studies, resulting in improved diuresis, natriuresis, and vasodilation (28). Therefore, several biological pathways exist in complement to the RAAS system, which are potential targets for neprilysin inhibitors.

RAAS inhibition has been of interest in HFpEF with ACEi and ARB showing improvement in functional capacity, symptoms, and hospital admissions (29,30), without evidence for reduction in mortality, and until now there remains a lack of proven regimens to guide management (31). Consequently, mortality and morbidity has been worse in this population compared to HFrEF (32,33).

In 2012, the multinational, phase 2 PARAMOUNT trial (34) compared sacubitril/valsartan with valsartan in patients with HFpEF ( $n=301)$. Patients were mostly NYHA class II but also included classes I and III. Decreased levels of NT-proBNP, surrogate marker of reduced left ventricular stress, has been correlated with improved outcomes in HF (35-37) and sacubitril/valsartan reduced NT-proBNP levels more than valsartan at a ratio of change 0.77 (95\% CI: 0.064-0.92; P=0.005) at 12 weeks. There was no change in LV mass, function, size as well as tricuspid regurgitant velocity or diastolic function. There was a significant reduction in left atrium volumes at week 36, suggestive of improved left ventricular filling pressures. Quality of life, hypotension, renal function or hyperkalemia was not statistically different between the arms. There was a significant improvement in NYHA class at 36 weeks. Overall sacubitril/valsartan was well tolerated and had a similar side-effect profile to valsartan. The effects on biomarkers and functional class were independent of the reduction in systolic blood pressure (38).

In PARAGON-HF (39), participants included NYHA II-IV ( $=4,822)$, LVEF $\geq 45 \%$ elevated natriuretic peptides. They were randomized to an ARNI or valsartan group with a target dose of sacubitril $97 \mathrm{mg}$ twice daily or valsartan $160 \mathrm{mg}$ twice daily. The primary composite outcome of total HF hospitalizations and cardiovascular death had a trend towards reduction in the ARNI group, HR 0.87 (95\% CI: $0.75-1.01 ; \mathrm{P}=0.06$ ). Quality of life at 8 months were 1.0 point lower in the ARNI arm (95\% CI: $0.0-2.1)$ by the KCCQ scores. Similarly, NYHA class improved with less deterioration in renal function, $1.4 \%$ and $2.7 \%$ respectively, HR 0.50 (95\% CI: 0.33-0.77). Further analysis of PARAGON-HF suggested a potential benefit for ARNI in patients with borderline or "mid-range" LVEF (40-50\%) $(31,40)$ as well as in females $(41)$.

Pooled analysis of PARAGON-HF and PARADIGM- 
HF to evaluate outcomes across the LVEF spectrum demonstrated that the benefit of ARNI was related to LVEF (treatment-by-continuous LVEF interaction $\mathrm{P}=0.02$ ). The effect had a U-shape relationship with benefit in those with reduced LVEF that diminished at a lower LVEF. This was mostly driven by HF hospitalizations. These benefits extended to higher LVEF range predominantly in women when compared to men (42).

A meta-analysis of 5 clinical trials evaluating RAAS inhibitors/ARNIs in HFpEF (43) showed that ARNI compared with controls reduced hospitalization for HF, OR 0.73 (95\% CI: 0.61-0.87) and compared with ARB, OR 0.80 (95\% CI: 0.71-0.91), not presenting heterogeneity between trials. Unfortunately, those trials failed to reveal statistical significance for cardiovascular or all-cause mortality between RAAS inhibition and placebo.

\section{Coronary artery disease (CAD)}

Natriuretic peptides increase after myocardial infarction (MI) have been linked to increased mortality (7). Animal models have shown that ARNI modulates natriuretic peptides thereby decreasing subsequent myocardial stiffness and remodeling, sparing of LVEF and mechanics, and decreasing LV dilation and fibrosis (44). In rat models with coronary artery ligation and secondary reduced LVEF, compared with enalapril therapy, sacubitril/valsartan modified CaMKII-p expression, upregulated expression of potassium channels, attenuated post-MI LV dysfunction and electrophysiologic remodeling reducing ventricular arrhythmia inducibility $(45,46)$.

PARADISE-MI (Prospective ARNI vs. ACE Inhibitor Trial to Determine Superiority in Reducing HF Events after MI, NCT02924727) aims to be the first large trial for sacubitril/valsartan use in acute coronary syndrome. Patients are randomized post-MI if LVEF $\leq 40 \%$ to start an ARNI or ramipril within 12 hours to 7 days after index event (26).

Similarly, data is lacking for neprilysin inhibition for stable CAD but post-hoc analysis of PARADIGM-HF provides some insights. Of the 8399 in PARADIGM-HF, $4796(57.1 \%)$ had prior MI, coronary revascularization, angiographic proof of $\mathrm{CAD}$ or stable/unstable angina. CAD related events were grouped into a "coronary" composite outcome of unstable angina, procedures for coronary revascularization, nonfatal MI or death from cardiovascular cause. In the ARNI arm, only death from cardiovascular cause was significantly reduced, but all other components of the composite were decreased in the ARNI compared to enalapril arm (47).

\section{Hypertension}

Multiple trials have shown the efficacy of ARNI in decreasing blood pressure. Sacubitril/valsartan provided larger blood pressure reduction in 1,328 participants as compared to a valsartan or placebo, without increase incidence of angioedema (48). The effectiveness and safety of sacubitril/valsartan has also been demonstrated when compared to placebo in a small population (49). There is yet to be a phase 3 trial for ARNI use in hypertension management for patients without cardiac involvement (7). The PARAMETER trial (50) randomized 454 geriatric participants with pulse pressures above $60 \mathrm{mmHg}$ to receive sacubitril/valsartan or olmesartan and those in the ARNI group required less additional antihypertensives, showed improved 24-hour ambulatory hypertension control and reduced central aortic blood pressures at 12 weeks.

\section{Cost-effectiveness}

Every country has individual willingness-to pay thresholds, so the high cost of sacubitril/valsartan may be prohibitive to health systems and patients in low to middle income countries while cost-effective in more expensive health care systems. In a comparison with enalapril in two separate trials in the United States, sacubitril/valsartan was found to have a cost per QALY gained of US $\$ 45,017$ and $\$ 50,959$, making it a cost-effective option in that health setting $(51,52)$. Sacubitril/valsartan can offset other high and prevalent costs in the health system such as HF hospitalizations. Furthermore, the current willingness-topay thresholds in the United States range from $\$ 50,000$ to $\$ 100,000$ per QALY gained but might be much lower in other countries. More affordable costs would likely expand the use of sacubitril/valsartan to other health systems.

\section{Safety and complications}

Sacubitril/valsartan has the benefits of dual RAAS and neprilysin inhibition without the life-threatening angioedema of omapatrilat because valsartan does not have the bradykinin and aminopeptidase inhibition that ACEi have. Consequently, by activating both systems, it has greater blood pressure reduction then ARB alone (48).

The robust reduction in blood pressure carries the 
potential complication of hypotension in some patients. In PARADIGM-HF (4), significant hypotension was minimized by excluding those with systolic blood pressure $<95 \mathrm{mmHg}$. Safety was further ensured by a 4-6 week runin period with both sacubitril/valsartan and enalapril at trial target doses. Throughout the trial and run-in period, significant hypotension affected $13 \%$ of patients (53). Those in the ANRI arm had more frequent symptomatic hypotension, but rarely significant enough to discontinue the medication. While symptomatic hypotension increased the likelihood of the primary study outcome, HR 2.63 (95\% CI: 2.21-3.13), the benefits of ARNI as compared to enalapril were similar in all groups including hypotensive patients. Consequently, only significant symptomatic hypotension leading to pre-syncope, syncope or other end organ damage should justify decreasing dosing of ARNI, ACEI, ARB, or MRA (26).

Although severe hyperkalemia was less frequent in the sacubitril/valsartan group in PARADIGM-HF trial, regular precautions for hyperkalemia and worsening renal function secondary to RAAS inhibition should be considered when prescribing sacubitril/valsartan. It is recommended to check creatinine and serum potassium 1-2 weeks after initiation and regularly thereafter (4).

The increase in bradykinin from neprilysin inhibition poses a theoretical risk for angioedema that was evident in omapatrilat trials, although studies with sacubitril/valsartan have not showed similar risk. Existing trials repeatedly show no significant difference in the rate of angioedema between ARNI and ACE inhibitors. Angioedema incidence is rare overall, and more trials are needed to strengthen these results $(24,54)$. In PARAGON-HF (33) angioedema was more common in the sacubitril/valsartan arm. Similar to other trials, the ARNI arm had more hypotension but less hyperkalemia. In PARADIGM-HF, enalapril was more likely to result in cough than sacubitril-valsartan.

Dementia has been a key topic of investigation. Because neprilysin inhibition may decrease degradation of amyloidbeta proteins in the brain (55), there remains a theoretical increased risk of Alzheimer's dementia (56). PARADIGMHF did not show any difference in dementia-related, memory, or cognitive adverse events (25), but the follow up time might have not been long enough. The ongoing PERSPECTIVE trial (NCT02884206) aims to address continued concern by using florbetapir- $18 \mathrm{~F}$ positron emission tomography imaging to the brain to track amyloid plaque deposition. It will also utilize a comprehensive set of cognitive testing while comparing sacubitril/valsartan against valsartan in HFpEF.

Cancer risk has also been postulated since by facilitating the metabolism of mitogenic peptides, neprilysin may function as a tumor cell proliferation checkpoint, particularly in prostate (57), breast (58) and other cancers $(7,59)$. It is likely that longer follow up might be necessary to show the clinical implications of neprilysin inhibition outside the cardiovascular system (7).

\section{Future directions and ongoing trials}

Most trials have focused on NYHA class II and III. The 60 patients with NYHA class IV HF only represented $<1 \%$ of PARADIGM enrollment (4). It also excluded those with low GFR, lower systolic blood pressure and higher NTproBNP, all of which are markers of poor prognosis and are highly prevalent in class IV patients. The HFN-LIFE trial (NCT02816736) is an ongoing randomized, doubleblind trial that compares sacubitril/valsartan with valsartan in $400 \mathrm{HFrEF}$ patients with advanced NYHA class IV HFrEF.

The benefits of ARNI in reverse cardiac remodeling are actively being investigated. PROVE-HF (NCT02887183) is an open label trial of NYHA class II-IV HFrEF patients, and will correlate NT pro-BNP with static and dynamic echocardiographic parameters. EVALUATE-HF (NCT02874794) will compare aortic impedance, among other echocardiographic parameters in patients with NYHA class I-III HFrEF randomized to sacubitril/valsartan or enalapril. The PRIME trial (NCT02687932) will evaluate reduction in functional mitral regurgitation in HF patients on sacubitril/valsartan versus valsartan alone.

Systemic effects and benefits are of equal concern. Renal function and albuminuria will be evaluated between ARNI and irbesartan in the UK HARP-III trial (ISRCTN 11958993). Mean pulmonary arterial pressures are being longitudinally measured by the CardioMEMS device in HFrEF patients on sacubitril/valsartan in the PARENT trial (NCT02788656), compared to standard of care.

Sacubitril/valsartan's benefits in class II-IV HF have led to multiple trials exploring the utility of ARNI at all stages of HF. The PARABLE trial evaluates the ability of sacubitril/valsartan to attenuate left atrial volume index in patients not yet diagnosed with HF or left ventricular dysfunction. Optimal timing to initiate sacubitril/ valsartan will be evaluated in the TRANSITION trial (NCT02661217), randomizing subjects for inpatient or outpatient initiation (day 1-14 post-discharge). 
As described above, PARADISE-MI will provide information in patients post MI with LVEF. The SILICOFCM trial (60) (NCT03832660), will provide insights into the effect of sacubitril/valsartan in patients with nonobstructive hypertrophic cardiomyopathy. Functional outcomes are equally important such as quality of life, symptom burden and exercise capacity, all of which will be measured in the PARALLAX study comparing sacubitril/valsartan with standard of care (61).

The effect of neprilysin inhibition on sleep-wakefulness is being addressed in 2 current trials. AWAKE-HF trial (NCT02970669) will compare sacubitril/valsartan with enalapril on objective daytime activity by wrist worn accelerometer. ENTRESTO-SAS (NCT02916160) seeks to determine if sacubitril/valsartan therapy for HF patients with sleep apnea syndrome may improve apnea-hypopnea indices (7).

\section{Conclusions}

Sacubitril/valsartan has proven to be an effective and potent addition to the HFrEF arsenal, with safety comparable to ACEI and ARB. In HFpEF, it improves quality of life, particularly in women and in patients with borderline ejection fraction, with no effect on mortality. Future studies will elucidate promising benefits in the fields of cardiovascular disease.

\section{Acknowledgments}

Funding: None.

\section{Footnote}

Provenance and Peer Review: This article was commissioned by the Guest Editors (Dr. Debabrata Mukherjee and Dr. Jose B. Cruz Rodriguez) for the series "Heart Failure Update and Advances in 2021" published in Annals of Translational Medicine. The article was sent for external peer review organized by the Guest Editors and the editorial office.

Reporting Checklist: The authors have completed the Narrative Review reporting checklist. Available at http:// dx.doi.org/10.21037/atm-20-4038

Conflicts of Interest: All authors have completed the ICMJE uniform disclosure form (available at http://dx.doi. org/10.21037/atm-20-4038). The series "Heart Failure Update and Advances in 2021" was commissioned by the editorial office without any funding or sponsorship. JBCR served as the unpaid Guest Editor of the series. The authors have no other conflicts of interest to declare.

Ethical Statement: The authors are accountable for all aspects of the work in ensuring that questions related to the accuracy or integrity of any part of the work are appropriately investigated and resolved.

Open Access Statement: This is an Open Access article distributed in accordance with the Creative Commons Attribution-NonCommercial-NoDerivs 4.0 International License (CC BY-NC-ND 4.0), which permits the noncommercial replication and distribution of the article with the strict proviso that no changes or edits are made and the original work is properly cited (including links to both the formal publication through the relevant DOI and the license). See: https://creativecommons.org/licenses/by-nc-nd/4.0/.

\section{References}

1. Ponikowski P, Anker SD, AlHabib KF, et al. Heart failure: preventing disease and death worldwide. ESC Heart Fail 2014;1:4-25.

2. Mozaffarian D, Benjamin EJ, Go AS, et al. Heart disease and stroke statistics-2016 update: a report from the American Heart Association. Circulation 2016;133:e38-360.

3. Lam CS, Donal E, Kraigher-Krainer E, et al. Epidemiology and clinical course of heart failure with preserved ejection fraction. Eur J Heart Fail 2011;13:18-28.

4. McMurray JJ, Packer M, Desai AS, et al. Angiotensinneprilysin inhibition versus enalapril in heart failure. $\mathrm{N}$ Engl J Med 2014;371:993-1004.

5. Martinez-Rumayor A, Richards AM, Burnett JC, et al. Biology of the natriuretic peptides. Am J Cardiol 2008;101:3-8.

6. Daniels LB, Maisel AS. Natriuretic peptides. J Am Coll Cardiol 2007;50:2357-68.

7. Riddell E, Vader JM. Potential expanded indications for neprilysin inhibitors. Curr Heart Fail Rep 2017;14:134-45.

8. Maric C, Zheng W, Walther T. Interactions between angiotensin II and atrial natriuretic peptide in renomedullary interstitial cells: the role of neutral endopeptidase. Nephron Physiol 2006;103:149-56.

9. Kuhn M. Molecular physiology of natriuretic peptide signalling. Basic Res Cardiol 2004;99:76-82. 
10. Potter LR, Abbey-Hosch S, Dickey DM. Natriuretic peptides, their receptors, and cyclic guanosine monophosphate-dependent signaling functions. Endocr Rev 2006;27:47-72.

11. Gardner DG, Chen S, Glenn DJ, et al. Molecular biology of the natriuretic peptide system: implications for physiology and hypertension. Hypertension 2007;49:419-26.

12. Rouleau JL, Pfeffer MA, Stewart DJ, et al. Comparison of vasopeptidase inhibitor, omapatrilat, and lisinopril on exercise tolerance and morbidity in patients with heart failure: IMPRESS randomised trial. Lancet 2000;356:615-20.

13. Packer M, Califf RM, Konstam MA, et al. Comparison of omapatrilat and enalapril in patients with chronic heart failure: the Omapatrilat Versus Enalapril Randomized Trial of Utility in Reducing Events (OVERTURE). Circulation 2002;106:920-6.

14. Kostis JB, Packer M, Black HR, et al. Omapatrilat and enalapril in patients with hypertension: the Omapatrilat Cardiovascular Treatment vs. Enalapril (OCTAVE) trial. Am J Hypertens 2004;17:103-11.

15. SOLVD Investigators, Yusuf S, Pitt B, et al. Effect of enalapril on survival in patients with reduced left ventricular ejection fractions and congestive heart failure. N Engl J Med 1991;325:293-302.

16. SOLVD Investigators, Yusuf S, Pitt B, et al. Effect of enalapril on mortality and the development of heart failure in asymptomatic patients with reduced left ventricular ejection fractions. N Engl J Med 1992;327:685-91.

17. Swedberg K, Kjekshus J. Effects of enalapril on mortality in severe congestive heart failure: results of the Cooperative North Scandinavian Enalapril Survival Study (CONSENSUS). Am J Cardiol 1988;62:60A-66A.

18. McMurray JJ, Packer M, Desai AS, et al. Dual angiotensin receptor and neprilysin inhibition as an alternative to angiotensin-converting enzyme inhibition in patients with chronic systolic heart failure: rationale for and design of the Prospective comparison of ARNI with ACEI to Determine Impact on Global Mortality and morbidity in Heart Failure trial (PARADIGM-HF). Eur J Heart Fail 2013;15:1062-73.

19. McMurray JJ, Packer M, Desai AS, et al. Baseline characteristics and treatment of patients in prospective comparison of ARNI with ACEI to determine impact on global mortality and morbidity in heart failure trial (PARADIGM-HF). Eur J Heart Fail 2014;16:817-25.

20. Packer M, McMurray JJ, Desai AS, et al. Angiotensin receptor neprilysin inhibition compared with enalapril on the risk of clinical progression in surviving patients with heart failure. Circulation 2015;131:54-61.

21. Okumura N, Jhund PS, Gong J, et al. Effects of sacubitril/ valsartan in the PARADIGM-HF trial (Prospective Comparison of ARNI with ACEI to Determine Impact on Global Mortality and Morbidity in Heart Failure) according to background therapy. Circ Heart Fail 2016;9:e003212.

22. Martens P, Beliën H, Dupont M, et al. The reverse remodeling response to sacubitril/valsartan therapy in heart failure with reduced ejection fraction. Cardiovasc Ther 2018;36:e12435.

23. Almufleh A, Marbach J, Chih S, et al. Ejection fraction improvement and reverse remodeling achieved with Sacubitril/Valsartan in heart failure with reduced ejection fraction patients. Am J Cardiovasc Dis 2017;7:108-13.

24. Velazquez EJ, Morrow DA, DeVore AD, et al. Angiotensin-neprilysin inhibition in acute decompensated heart failure. N Engl J Med 2019;380:539-48.

25. Solomon SD, Claggett B, McMurray JJ, et al. Combined neprilysin and renin-angiotensin system inhibition in heart failure with reduced ejection fraction: a meta-analysis. Eur J Heart Fail 2016;18:1238-43.

26. Leong DP, McMurray JJ, Joseph PG, et al. From ACE Inhibitors/ARBs to ARNIs in Coronary Artery Disease and Heart Failure (Part 2/5). J Am Coll Cardiol 2019;74:683-98.

27. Krüger M, Kötter S, Grützner A, et al. Protein kinase $G$ modulates human myocardial passive stiffness by phosphorylation of the titin springs. Circ Res 2009;104:87-94.

28. Gori M, D'Elia E, Senni M. Sacubitril/valsartan therapeutic strategy in HFpEF: Clinical insights and perspectives. Int J Cardiol 2019;281:158-65.

29. Cleland JG, Tendera M, Adamus J, et al. The perindopril in elderly people with chronic heart failure (PEP-CHF) study. Eur Heart J 2006;27:2338-45.

30. Yusuf S, Pfeffer MA, Swedberg K, et al. Effects of candesartan in patients with chronic heart failure and preserved left-ventricular ejection fraction: the CHARMPreserved Trial. Lancet 2003;362:777-81.

31. McMurray JJ, Adamopoulos S, Anker SD, et al. ESC Guidelines for the diagnosis and treatment of acute and chronic heart failure 2012: The Task Force for the Diagnosis and Treatment of Acute and Chronic Heart Failure 2012 of the European Society of Cardiology. Developed in collaboration with the Heart 
Failure Association (HFA) of the ESC. Eur Heart J 2012;33:1787-847.

32. Aurigemma GP, Gaasch WH. Diastolic heart failure. N Engl J Med 2004;351:1097-105.

33. Shah RV, Desai AS, Givertz MM. The effect of reninangiotensin system inhibitors on mortality and heart failure hospitalization in patients with heart failure and preserved ejection fraction: a systematic review and metaanalysis. J Card Fail 2010;16:260-7.

34. Solomon SD, Zile M, Pieske B, et al. The angiotensin receptor neprilysin inhibitor LCZ696 in heart failure with preserved ejection fraction: a phase 2 double-blind randomised controlled trial. Lancet 2012;380:1387-95.

35. Masson S, Latini R, Anand IS, et al. Prognostic value of changes in $\mathrm{N}$-terminal pro-brain natriuretic peptide in Val-HeFT (Valsartan Heart Failure Trial). J Am Coll Cardiol 2008;52:997-1003.

36. Komajda M, Carson PE, Hetzel S, et al. Factors associated with outcome in heart failure with preserved ejection fraction: findings from the Irbesartan in Heart Failure with Preserved Ejection Fraction Study (I-PRESERVE). Circ Heart Fail 2011;4:27-35.

37. Cleland JG, Taylor J, Tendera M. Prognosis in heart failure with a normal ejection fraction. $\mathrm{N}$ Engl J Med 2007;357:829-30.

38. Jhund PS, Claggett B, Packer M, et al. Independence of the blood pressure lowering effect and efficacy of the angiotensin receptor neprilysin inhibitor, LCZ696, in patients with heart failure with preserved ejection fraction: an analysis of the PARAMOUNT trial. Eur J Heart Fail 2014;16:671-7.

39. Solomon SD, McMurray JJ, Anand IS, et al. Angiotensinneprilysin inhibition in heart failure with preserved ejection fraction. N Engl J Med 2019;381:1609-20.

40. Lam CS, Solomon SD. The middle child in heart failure: heart failure with mid-range ejection fraction (40-50\%). Eur J Heart Fail 2014;16:1049-55.

41. McMurray JJ, Jackson AM, Lam CS, et al. Effects of sacubitril-valsartan versus valsartan in women compared with men with heart failure and preserved ejection fraction: insights from PARAGON-HF. Circulation 2020;141:338-51.

42. Solomon SD, Vaduganathan M, Claggett BL, et al. Sacubitril/valsartan across the spectrum of ejection fraction in heart failure. Circulation 2020;141:352-61.

43. Kuno T, Ueyama H, Fujisaki T, et al. Meta-Analysis Evaluating the Effects of Renin-Angiotensin-Aldosterone System Blockade on Outcomes of Heart Failure
With Preserved Ejection Fraction. Am J Cardiol 2020;125:1187-93.

44. von Lueder TG, Wang BH, Kompa AR, et al. Angiotensin receptor neprilysin inhibitor LCZ696 attenuates cardiac remodeling and dysfunction after myocardial infarction by reducing cardiac fibrosis and hypertrophy. Circ Heart Fail 2015;8:71-8.

45. Chang PC, Lin SF, Chu Y, et al. LCZ696 Therapy Reduces Ventricular Tachyarrhythmia Inducibility in a Myocardial Infarction-Induced Heart Failure Rat Model. Cardiovasc Ther 2019;2019:6032631.

46. Chang PC, Wo HT, Lee HL, et al. Sacubitril/Valsartan Therapy Ameliorates Ventricular Tachyarrhythmias Inducibility in a Rabbit Myocardial Infarction Model. J Card Fail 2020;26:527-37.

47. Mogensen UM, Køber L, Kristensen SL, et al. The effects of sacubitril/valsartan on coronary outcomes in PARADIGM-HF. Am Heart J 2017;188:35-41.

48. Ruilope LM, Dukat A, Böhm M, et al. Blood-pressure reduction with LCZ696, a novel dual-acting inhibitor of the angiotensin II receptor and neprilysin: a randomised, double-blind, placebo-controlled, active comparator study. Lancet 2010;375:1255-66.

49. Kario K, Sun N, Chiang FT, et al. Efficacy and safety of LCZ696, a first-in-class angiotensin receptor neprilysin inhibitor, in Asian patients with hypertension: a randomized, double-blind, placebo-controlled study. Hypertension 2014;63:698-705.

50. Williams B, Cockcroft JR, Kario K, et al. Effects of Sacubitril/Valsartan Versus Olmesartan on Central Hemodynamics in the Elderly With Systolic Hypertension: The PARAMETER Study. Hypertension 2017:69:411-20.

51. King JB, Shah RU, Bress AP, et al. Cost-effectiveness of sacubitril-valsartan combination therapy compared with enalapril for the treatment of heart failure with reduced ejection fraction. JACC Heart Fail 2016;4:392-402.

52. Gaziano TA, Fonarow GC, Claggett B, et al. Costeffectiveness analysis of sacubitril/valsartan vs. enalapril in patients with heart failure and reduced ejection fraction. JAMA Cardiol 2016;1:666-72.

53. Vardeny O, Claggett B, Kachadourian J, et al. Incidence, predictors, and outcomes associated with hypotensive episodes among heart failure patients receiving sacubitril/ valsartan or enalapril: the PARADIGM-HF trial (prospective comparison of angiotensin receptor neprilysin inhibitor with angiotensin-converting enzyme inhibitor to determine impact on global mortality and morbidity in heart failure). Circ Heart Fail 2018;11:e004745. 
54. Shi V, Senni M, Streefkerk H, et al. Angioedema in heart failure patients treated with sacubitril/valsartan (LCZ696) or enalapril in the PARADIGM-HF study. Int J Cardiol 2018;264:118-23.

55. Campbell DJ. Long-term neprilysin inhibitionimplications for ARNIs. Nat Rev Cardiol 2017;14:171-86.

56. Langenickel TH, Tsubouchi C, Ayalasomayajula S, et al. The effect of LCZ696 (sacubitril/valsartan) on amyloid- $\beta$ concentrations in cerebrospinal fluid in healthy subjects. Br J Clin Pharmacol 2016;81:878-90.

57. Shen R, Sumitomo M, Dai J, et al. Androgen-induced growth inhibition of androgen receptor expressing androgen-independent prostate cancer cells is mediated by increased levels of neutral endopeptidase. Endocrinology 2000;141:1699-704.

58. Stephen HM, Khoury RJ, Majmudar PR, et al. Epigenetic

Cite this article as: Cruz Rodriguez $\mathrm{JB}, \mathrm{Cu} \mathrm{C}$, Siddiqui T. Narrative review in the current role of angiotensin receptorneprilysin inhibitors. Ann Transl Med 2021;9(6):518. doi: 10.21037/atm-20-4038 suppression of neprilysin regulates breast cancer invasion. Oncogenesis 2016;5:e207.

59. Terauchi M, Kajiyama H, Shibata K, et al. Anti-progressive effect of neutral endopeptidase 24.11 (NEP/CD10) on cervical carcinoma in vitro and in vivo. Oncology 2005;69:52-62.

60. Tafelmeier M, Baessler A, Wagner S, et al. Design of the SILICOFCM study: Effect of sacubitril/valsartan vs. lifestyle intervention on functional capacity in patients with hypertrophic cardiomyopathy. Clin Cardiol 2020;43:430-40.

61. Wachter R, Shah SJ, Cowie MR, et al. Angiotensin receptor neprilysin inhibition versus individualized RAAS blockade: design and rationale of the PARALLAX trial. ESC Heart Fail 2020;7:856-64. 